\title{
The Dynamics of a Rapidly Escaping Atmosphere: Applications to the Evolution of Earth and Venus
}

\author{
ANDREW J. WATSON, THOMAS M. DONAHUE, AND JAMES C. G. WAL KER \\ Space Physics Research Laboratory, University of Michigan, Ann Arbor, Michigan 48109
}

Received April 17, 1981; revised September 22, 1981

\begin{abstract}
A simple, idealized model for the rapid escape of a hydrogen thermosphere provides some quantitative estimates for the energy-limited flux of escaping particles. The model assumes that the atmosphere is "tightly bound" by the gravitational field at lower altitudes, that diffusion through the lower atmosphere does not limit the flux, and that the main source of heating is solar euv. Rather low thermospheric temperatures are typical of such escape and a characteristic minimum develops in the temperature profile as the escape flux approaches its maximum possible value. The flux is limited by the amount of euv energy absorbed, which is in turn controlled by the radial extent of the thermosphere. Regardless of the amount of hydrogen in the thermosphere, the low temperatures accompanying rapid escape limit its extent, and thus constrain the flux. Applied to the Earth and Venus, the results suggest that the escape of hydrogen from these planets would have been energy-limited if their primordial atmospheres contained total hydrogen mixing ratios exceeding a few percent. Hydrogen and deuterium may have been lost in bulk, but heavier elements would have remained in the atmosphere. These results place constraints on hypotheses for the origin of the planets and their subsequent evolution.
\end{abstract}

\section{INTRODUCTION}

Early in their evolution, the atmospheres of the terrestrial planets probably contained considerably more hydrogen than they do today (Holland, 1962; Walker, 1977; Ringwood, 1979; Pollack and Yung, 1980). Exactly how much more is still a subject of debate, but some theories advanced for the origin of the Earth envisage the loss of vast quantities of hydrogen and heavier volatiles at an early stage (Ringwood, 1975, 1979; Sekiya et al., 1980a,b). On Venus, hydrogen escape following on a "runaway greenhouse" event may explain the low abundance of water observed on that planet (Dayhoff et al., 1967; Ingersoll, 1969; Walker, 1975; Pollack and Black, 1979). In this paper we examine the dynamics of rapid hydrogen escape in an attempt to place constraints on these theories.

We consider the case of a planetary atmosphere after the solar nebula has dissipated. We assume an extensive thermosphere of molecular or atomic hydrogen that contains a negligible amount of dust. Energy input to the thermosphere is dominated by the absorption of solar euv radiation. On the terrestrial planets today, the escape of light gases may occur via thermal evaporation (Jeans' escape) or by any of several mechanisms involving charged particles (Hunten and Donahue, 1976). These latter, nonthermal mechanisms are of comparable importance to Jeans' escape on the Earth, and are the major loss process on Venus. However, nonthermal processes are not included in our treatment; the hydrodynamic escape which we discuss is essentially a thermal process. Hydrodynamic escape differs from gas-kinetic evaporation in that in some circumstances a substantial fraction of the entire thermospheric energy budget may be used to power the escape of gas. It is these circumstances in which we are most interested, and we expect hydrodynamic expansion to be the dominant loss process under these conditions.

The process of Jeans' escape has been extensively studied (see, for example, 
Jeans, 1925; Chamberlain, 1963, and references given there). The rate of loss is found to be sensitively dependent on the escape parameter, $\lambda_{\mathrm{J}}$, defined by

$$
\lambda_{\mathrm{J}}=G M m / k T_{\mathrm{J}} r_{\mathrm{J}} .
$$

Here $G$ is the gravitational constant, $M$ is the mass of the planet, $m$ is the mass of the escaping particles, and $k$ is Boltzmann's constant. $T_{\mathrm{J}}$ and $r_{\mathrm{J}}$ are respectively the temperature and radius of the exobase. Provided the escape rate is slow enough that the energy lost with the escaping particles does not appreciably affect temperatures near the exobase, values for $T_{\mathrm{J}}$ and $r_{\mathrm{J}}$ may be found by treating the atmosphere as static (Chamberlain, 1962; McElroy, 1968). When applied to a dense hydrogen thermosphere on one of the inner planets, this technique fails completely, as Gross (1972) has shown; one obtains exospheric temperatures in excess of $10,000^{\circ} \mathrm{K}$ and values of $\lambda_{J}$ less than one. But if the escape parameter is less than 1.5 (or 2.5 for a diatomic gas) the internal energy of the gas is greater than the depth of the gravitational potential well in which it is situated. The gas should therefore flow off the planet in bulk rather than merely evaporate from the exobase (Öpik, 1963; Shklovskii, 1951). Jeans' formula is not applicable under these circumstances and a hydrodynamic treatment becomes necessary.

The hydrodynamic escape of a light gas from a planetary atmosphere is a process similar in many respects to the supersonic flow of plasma from the Sun (Parker, 1963). The solar wind approach to the planetary problem was developed by Gross (1974), and applied by Hunten (1979) to the dissipation of a primordial atmosphere on Mars. Gross and Hunten both used a polytrope law to model the escaping gas, according to which the temperature varies as $n^{\alpha-1}$, where $n$ is the number density and $\alpha$ is a constant index. Use of a polytrope greatly simplifies the solution of the hydrodynamic equations (Parker, 1963). Furthermore, good first-order results are obtained for the case of the solar wind, where essentially all of the energy input is near the base of the atmosphere and the temperature declines monotonically outward from the Sun. For a planetary atmosphere where there is heating from in situ absorption as well as from the lower boundary, the use of the polytrope representation is questionable. Effectively, its use imposes on the energy input a particular spatial dependence (Hunten, 1979), which does not necessarily correspond at all with the heating in a planetary atmosphere. For this reason we do not attempt to use the polytrope approximation.

The rapid escape of matter from an atmosphere requires a concomitant expenditure of energy to lift the gas in the gravitational field (McElroy, 1974). Let us suppose that this energy is supplied by euv heating at a rate $S$ ergs $\mathrm{cm}^{-2} \mathrm{sec}^{-1}$, where $S$ has been suitably averaged over a sphere and corrected for the less-than-unity efficiency of euv heating. Let the radius of the planet be $r_{0}$ and the ultraviolet be mostly absorbed near $r_{1}$, the level where the optical depth is unity. Equating the energy input to the energy of escape then yields

$$
F=S r_{1}{ }^{2} r_{0} / G M m
$$

for the escape flux of particles per steradian per second. An upper limit to the escape rate is thus immediately obtained, provided the value of $r_{1}$ can be specified. However, fixing $r_{1}$ is not such a simple matter. Superficially, we might expect that if the density at the base of the thermosphere is increased, the position of unity optical depth will move outward, allowing progressively higher escape rates. In the following section we set up a simple model for the thermosphere which allows us to solve simultaneously for the value of $r_{1}$ and the maximum escape flux associated with it, as a function of the incident euv. We find that under many conditions the escape flux is constrained regardless of the amount of hydrogen which is present. In the third section, results of numerical solutions to the hydrodynamic equations are displayed and 
compared with the simple model. In the last section some applications to the evolution of Earth and Venus are discussed.

\section{THE ENERGY-LIMITED FLUX}

Consider a dynamically expanding, nonviscous gas of constant molecular weight, in which the pressure is isotropic. The steady-state equations of mass, momentum, and energy conservation are respectively,

$$
\begin{gathered}
\nabla \cdot(n \mathbf{u})=0, \\
m n(\mathbf{u} \cdot \nabla) \mathbf{u}+\nabla(n k T)=n m \mathbf{g}, \\
\nabla \cdot(\kappa \nabla T)=\nabla \cdot\left[\frac{5}{2} n k T \mathbf{u}\right. \\
\left.+n\left(m u^{2} / 2\right) \mathbf{u}\right]-n m \mathbf{g} \cdot \mathbf{u}-q,
\end{gathered}
$$

where $\mathbf{u}$ is the bulk velocity of the gas, $\mathbf{g}$ is the acceleration of gravity, $\kappa$ is the thermal conductivity, and $q$ is the volume heating rate. The factor $\frac{5}{2}$ in (5) is specific to a monatomic gas and would be replaced by $\frac{7}{2}$ for diatomic particles.

The equations are identical to those used to model the solar wind, with the exception that in those studies the heating term $q$ is usually assumed to be zero. The equations may be considerably reduced by the use of dimensionless variables (Chamberlain, 1961; Noble and Scarf, 1963). Following these authors' treatments we introduce an arbitrary reference temperature, $T_{0}$, and define temperature, velocity, and position variables as follows

$$
\begin{aligned}
\tau & =T / T_{0}, \\
\Psi & =m u^{2} / k T_{0}, \\
\lambda & =G M m / k T_{0} r .
\end{aligned}
$$

(Note that whereas $\lambda$ is here defined in terms of an arbitrary temperature, the escape parameter $\lambda_{J}$ is defined by Eq. (1) specifically in terms of the exobase temperature.) The thermal conductivity is parameterized by

$$
\kappa=\kappa_{0} \tau^{s},
$$

where $s$ is about 0.7 for a neutral gas (Banks and Kockarts, 1973). Assuming radial sym- metry, (3) may be immediately integrated to give

$$
F=n u r^{2},
$$

while (4) and (5) may be reduced to the following expressions (Noble and Scarf, 1963, Eqs. (8) and (9)).

$$
\begin{array}{r}
(1-\tau / \Psi) d \Psi / d \lambda \\
=2(1-2 \tau / \lambda-d \tau / d \lambda) \\
\left(\kappa_{0} G M m / k^{2} T_{0} F\right) \tau^{8}(d \tau / d \lambda) \\
=\epsilon+\lambda-5 \tau / 2-\Psi / 2 \\
\epsilon=\epsilon_{\infty}-\left(1 / F k T_{0}\right) \int_{\mathrm{r}}^{\infty} q r^{2} d r
\end{array}
$$

where we have been careful to retain $q \neq 0$. The quantity $\epsilon$ is the outward energy flow per escaping particle, expressed in units of $k T_{0} . \epsilon_{\infty}$ is the energy flow at infinity. Though $\epsilon$ may in general be either positive or negative, $\epsilon_{\infty}$ cannot be less than zero unless there exists an energy source at infinity (Parker, 1964a). For the special case where $\epsilon_{\infty}=0$, the outward flow of energy is just sufficient to lift the gas out of the gravitational field, leaving no excess to be conducted or advected at very large distances.

To apply these equations to a dense thermosphere of hydrogen we make the following assumptions:

(1) At a lower boundary located some distance above the homopause the temperature is fixed at $T_{0}$, thus $\tau\left(\lambda_{0}\right)=1$. The lower boundary is sufficiently far from the homopause that the mixing ratios of heavier gases are much smaller than that of hydrogen, which therefore dominates at all higher altitudes. We treat the case where the atmosphere is "tightly bound" by gravity at the lower boundary; i.e., $\lambda_{0} \geq 10$ (Parker, $1964 a, b)$. For $H$ atoms on Earth this condition is satisfied provided $\mathrm{T}_{0}<750^{\circ} \mathrm{K}$; for $\mathrm{H}_{2}$ molecules the equivalent condition is $T_{0}<$ $1500^{\circ} \mathrm{K}$. For most applications a reasonable value for $T_{0}$ would be the skin temperature of the planet, i.e., $200-300^{\circ} \mathrm{K}$ for Venus or Earth.

(2) The atmosphere is sufficiently dense that the optical depth of the lower boundary 
to euv is much greater than one. For the present we further simplify the heating by assuming that all the euv is absorbed in a narrow region at $r_{1}$, where the optical depth is unity. Above this level, Eq. (9b) then reduces to $\epsilon=\epsilon_{\infty}$. Below $r_{1}$ the equation becomes

$$
\epsilon=\epsilon_{\infty}-S r_{1}{ }^{2} / F k T_{0},
$$

corresponding to complete absorption of all the available energy. Since there is little energy deposited above $r_{1}$ and the gas is continually expanding, the temperature declines as $r \rightarrow \infty$.

(3) The pressure at large distances declines toward zero. Because of this the gas must expand according to a "critical" solution of the hydrodynamic equation (Parker, 1963). At the "sonic level," $r_{\mathrm{s}}$, the velocity passes smoothly from subsonic flow (defined by the condition $\Psi<\tau$ ) to supersonic flow, $\Psi>\tau$. We assume that $r_{1}<r_{\mathrm{s}}$. In the Appendix it is shown that this assumption implies

$$
\lambda_{1} / \tau_{1}>2 \text {, }
$$

which allows us to place limits on temperatures in the thermosphere. A justification of this assumption for the cases of interest is given in the Appendix. We note here that it is not necessarily valid if the atmosphere is very strongly heated by uv absorption. Under such conditions the assumption of a localized heating function may also break down.

We now focus our attention on the energy $\mathrm{Eq}$. (9). It is convenient to define the following dimensionless variables for the flux of escaping particles and the euv heating:

$$
\begin{aligned}
& \zeta=F \cdot\left(k^{2} T_{0} / \kappa_{0} G M m\right), \\
& \beta=S \cdot\left(G M m / k T_{0}^{2} \kappa_{0}\right) .
\end{aligned}
$$

Substituting (10) in (9b) and (9b) in (9a), the energy equation for the region below $r_{1}$ may now be written

$$
\begin{aligned}
\left(\tau^{s} / \zeta\right) d \tau / d \lambda=\lambda & -\beta /\left(\zeta \lambda_{1}{ }^{2}\right) \\
& +\epsilon_{\infty}-5 \tau / 2-\Psi / 2 .
\end{aligned}
$$

Well below the sonic level the velocity term is negligible. Near the lower boundary, $\lambda \gg$ $\tau$ from our first assumption, so the equation may be further simplified;

$$
\left(\tau^{8} / \zeta\right) d \tau / d \lambda \approx \lambda-\beta /\left(\zeta \lambda_{1}^{2}\right)+\epsilon_{\infty}
$$

Using this approximate equation we now show that only values of the escape flux below a certain limit will yield a physical solution. If the flux variable $\zeta$ is small enough, the r.h.s. of (15) will be negative for all values of $\lambda$ less than $\lambda_{0} . d \tau / d \lambda$ is then also negative, meaning that the temperature increases monotonically from the lower boundary out to $r_{1}$. A temperature profile resembling curve $A$ of Fig. 1 will then result. Under these conditions a portion of the energy deposited at $r_{1}$ is conducted down the temperature gradient and out of the lower boundary. But as $\zeta$ is allowed to increase, a minimum appears in the temperature profile, at a position given by

$$
\lambda \approx \beta /\left(\zeta \lambda_{1}^{2}\right)-\epsilon_{\infty}
$$

Conducted heat now flows into the minimum from both above and below, while the expansion of the gas in the gravitational field tends to cool this region. With further increases in the escape flux the minimum becomes progressively deeper. The maximum possible flux is obtained as the temperature tends to zero, as in curve B of Fig. 1. A still higher flux would yield negative temperatures and is therefore unrealistic. Physically, the escape flux is limited by the requirement that sufficient energy be conducted downward from $r_{1}$, where it is deposited, to the inner thermosphere, where it is required to offset the adiabatic cooling of the rapidly expanding gas.

Consider now the effect of allowing the value of $r_{1}$ to increase. The energy absorbed per steradian increases as $r_{1}^{2}$, but the greater distance between $r_{1}$ and the temperature minimum tends to impede the downward conduction of energy. If $r_{1}$ becomes too great the second effect domi- 


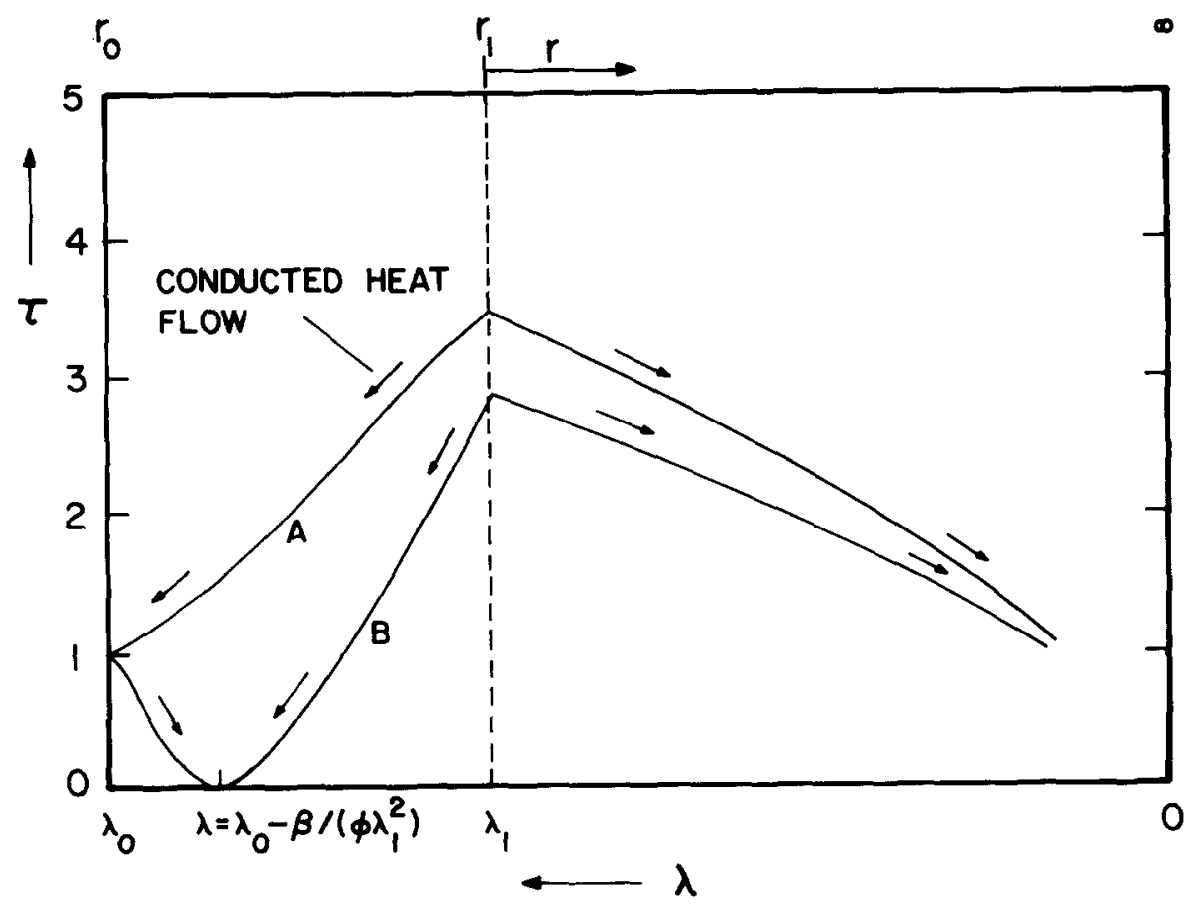

FIG. 1. Temperature profiles in a rapidly expanding thermosphere. Curve A: energy is conducted downward from $\lambda_{1}$ to $\lambda_{0}$ and upward from $\lambda_{1}$ to infinity. Curve B: at higher escape rates a minimum appears in the profile; the temperature at the minimum approaches zero as the flux approaches the energy-limited rate. Arrows show direction of conducted energy flow.

nates and the escape flux must "throttle down" for lack of energy.

In the Appendix we utilize Eq. (15) to obtain simultaneous equations for the energy-limited flux and $\lambda_{1}$, the value of the radial parameter at the level of energy deposition. The equations are derived by applying conditions at the temperature minimum. For the maximum flux case, both $\tau$ and $d \tau / d \lambda$ must be zero at that point. Relation (11) is used to set limits on the temperature at $r_{1}$. It should be noted that the energy "throttle" may not operate if this temperature rises continuously as $r_{1}$ increases [i.e., under conditions where (11) is not valid]. Such conditions require further study.

The equations for the maximum flux, $\zeta_{\mathrm{m}}$, and the associated value of $\lambda_{1}$ are

$$
\zeta_{\mathrm{m}}=\frac{2}{s+1}\left[\frac{\left(\lambda_{1} / 2\right)^{(8+1) / 2}+1}{\lambda-\lambda_{1}+\epsilon^{\prime}}\right]^{2},
$$

$$
\begin{aligned}
\lambda_{1}=\left(\frac{\beta}{\zeta_{m}}\right. & \left\{\lambda_{0}\right. \\
- & {\left.\left.\left[\frac{2}{(1+s) \zeta_{m}}\right]^{1 / 2}+\epsilon_{\infty}\right\}^{-1}\right)^{1 / 2}, }
\end{aligned}
$$

where $\epsilon^{\prime}$ in (16) is a positive constant defined in the Appendix.

The simultaneous solution of these equations for $\zeta_{m}$ and $\lambda_{1}$ must be accomplished by numerical means with specified values of $\beta$, $\lambda_{0}, \epsilon_{\infty}$, and $\epsilon^{\prime}$. However, we show in the Appendix that setting $\epsilon_{\infty}=\epsilon^{\prime}=0$ can result only in an overestimate of the flux. Since our main interest is to obtain an upper limit to the rate of escape we may justifiably neglect these terms. With this simplification, the solution for $\zeta_{m}$ as a function of $\beta$ is displayed in Fig. 2. Curves are shown for 5 values of $\lambda_{0}$. Figure 3 shows corresponding values for $r_{1} / r_{0}$ (which equals $\left.\lambda_{0} / \lambda_{1}\right)$. As an aid to the physical interpretation of these figures, Table I 


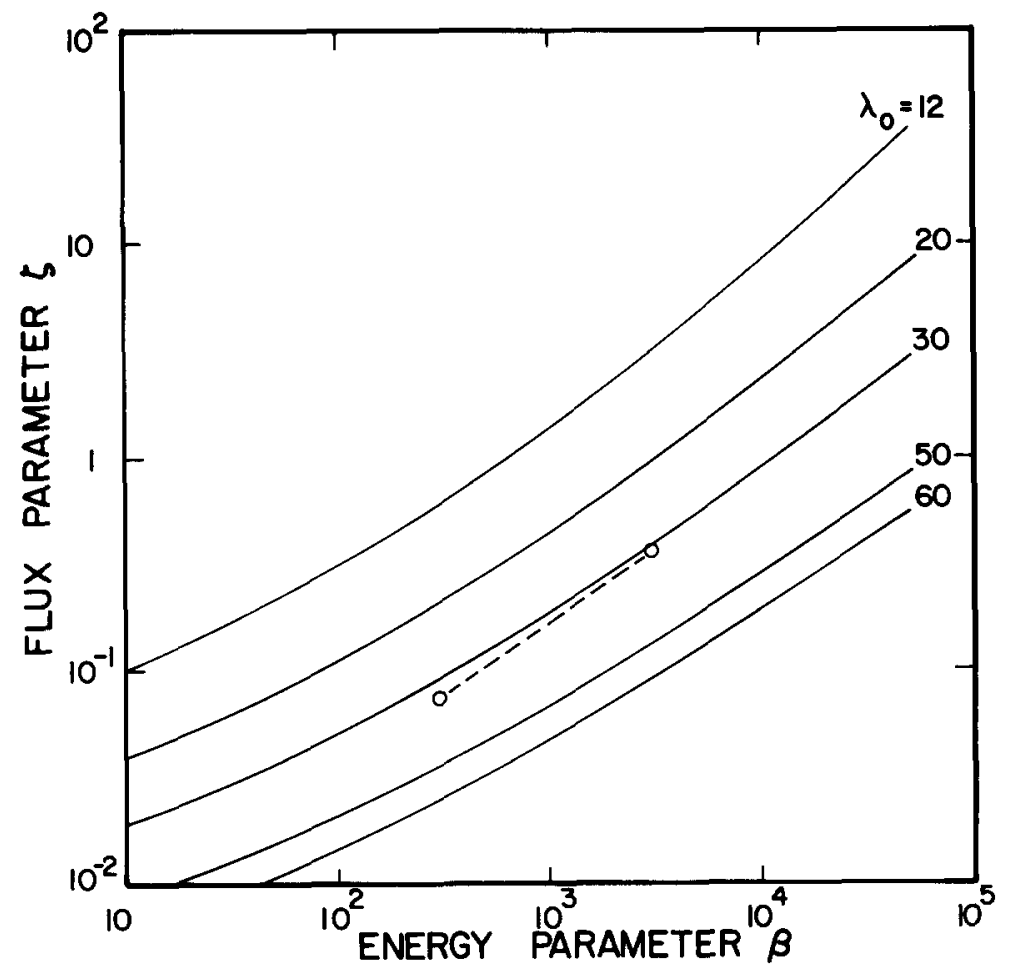

FIG. 2. Dependence of the flux variable $\zeta$ on the energy parameter $\beta$ for various values of $\lambda_{0}$ obtained by numerical solution of Eqs. (16) and (17) with $\epsilon_{\infty}=\epsilon^{\prime}=0$. Marked for comparison are results obtained by solution of the full hydrodynamic equations, for $\lambda_{0}=30$.

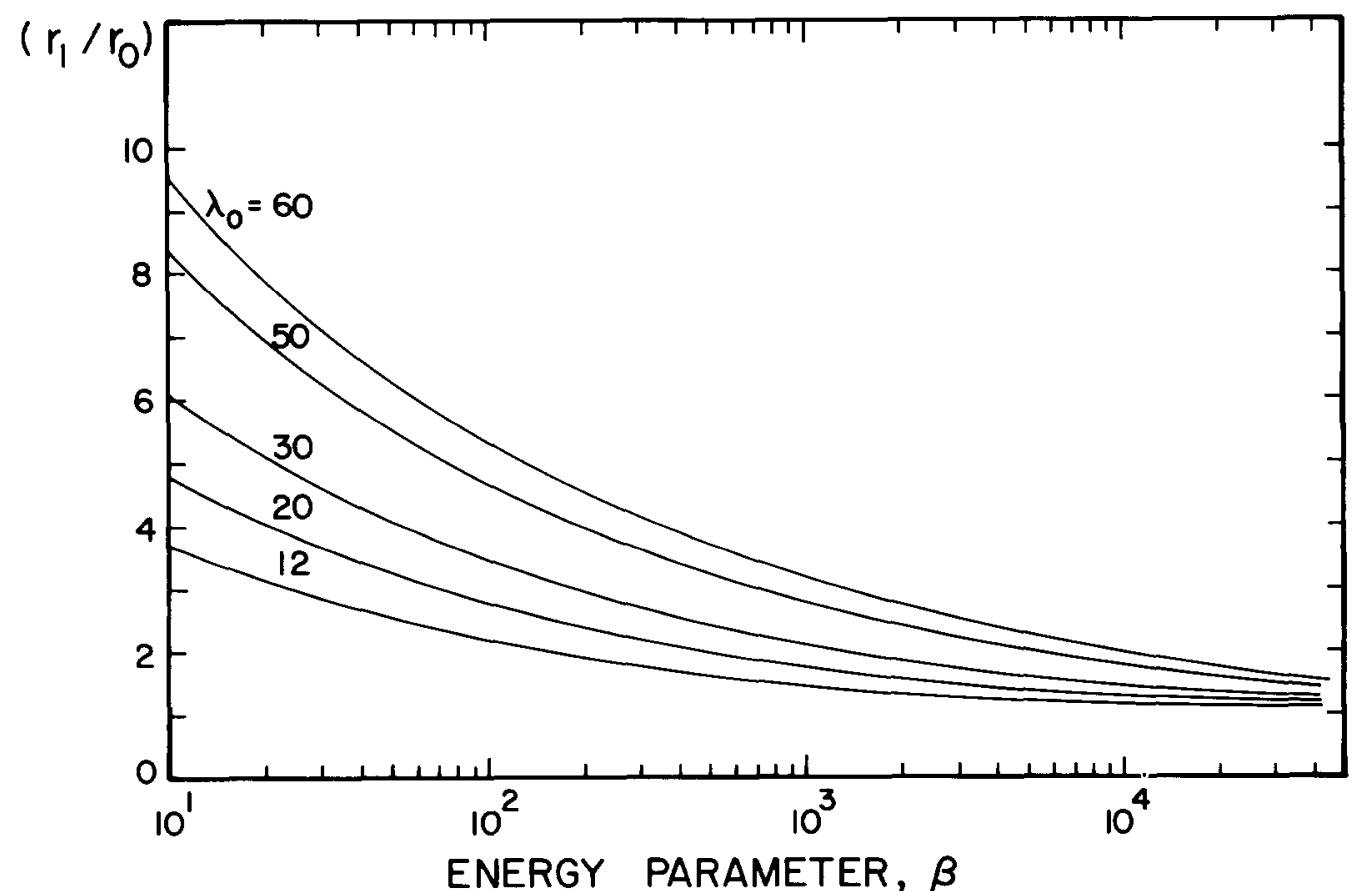

FIG. 3. Distance of unity optical depth in units of the planetary radius, as a function of $\beta$. Calculated from Eqs. 16 and 17. 
TABLE I

Typical Values of Dimensioniess Parameters FOR THE EARTH ${ }^{a}$

\begin{tabular}{ccc}
\hline $\begin{array}{c}\text { Dimensionless } \\
\text { parameter }\end{array}$ & $\begin{array}{c}\text { Value for } \\
\text { H atoms }\end{array}$ & $\begin{array}{c}\text { Value for } \\
\mathrm{H}_{2} \text { molecules }\end{array}$ \\
\hline$\lambda_{0}$ & 30.1 & 60.2 \\
$\zeta$ & 0.043 & 0.023 \\
$\beta$ & $4.5 \times 10^{3}$ & $9.6 \times 10^{3}$ \\
\hline
\end{tabular}

${ }^{a}$ We take $T_{0}=250^{\circ} \mathrm{K}, M=$ Earth's mass, $r_{0}=$

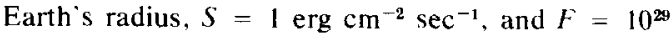
particles $\mathrm{sr}^{-1} \mathrm{sec}^{-1}$ (or $2.5 \times 10^{11} \mathrm{~cm}^{-2} \mathrm{sec}^{-1}$ at the surface).

shows some typical values of the dimensionless parameters $\lambda_{0}, \zeta$, and $\beta$ as applied to the Earth. The figures show that increasing the incident euv flux allows the maximum escape rate to rise but tends to decrease the "extent" of the atmosphere as measured by $r_{1} / r_{0}$. The escape flux thus increases less rapidly than the incident euv. The atmosphere is generally never so extensive that $r_{1}$ exceeds $10 r_{0}$.

Our analysis is not dependent on the density of the thermosphere, provided it is sufficient for all the euv flux to be absorbed. It follows that the escape flux and the extent of the atmosphere are constrained regardless of how much hydrogen is present near the homopause; the deep temperature minimum serves to decouple densities in the outer region from those near the lower boundary. (This property is more clearly illustrated by the numerical examples of the next section.)

\section{NUMERICAL CALCULATIONS}

The analytical treatment of the hydrodynamic equations is only possible under highly idealized conditions. We have employed numerical methods to obtain temperature, density, and velocity profiles under less restrictive assumptions for a few particular cases. Our method is a modification of the technique of Wang and Chang (1965), which they applied to the solar wind. Wang and Chang used trial-anderror methods to obtain the set of critical solutions which matched a boundary condition of zero temperature at infinity. In our study the subset of these solutions that match a given temperature at the lower boundary is found, again by trial-and-error integrations beginning at the sonic level. Each of these doubly matched solutions has a unique number density at the lower boundary and a unique escape flux.

Rather than perform the full heating calculation, we employ a mean absorption cross section, calculated as a weighted average over the solar euv spectrum. Above the sonic level, the heating term is neglected. This simplification is not quite as arbitrary as it may appear; at some distance from the planet the gas must become sufficiently tenuous that recombination processes can no longer keep up with photoionization; the gas then becomes progressively more ionized and the heating due to photoionization declines toward zero. For the numerical solutions studied this normally occurred in the neighborhood of the sonic level.

Figures 4, 5, and 6 show respectively temperature, number density and velocity profiles appropriate to a dense thermosphere of hydrogen atoms escaping from the Earth. The efficiency of euv heating is taken to be $15 \%$. The lower boundary is fixed at $130 \mathrm{~km}$ altitude with a temperature of $250^{\circ} \mathrm{K}$, giving a value of approximately 30 for $\lambda_{0}$. The "maximum flux" solution is approached as the number density at the lower boundary is progressively increased in curves $A$ through $E$. Figure 4 shows the development of a minimum in the temperature profile as the flux approaches its maximum allowed value. Note that temperatures are everywhere quite low. This is in marked contrast to the temperatures of tens of thousands of degrees which result if a hydrogen thermosphere is treated as static (Gross, 1972). As the energy-limited solution is approached, the density of the inner region becomes very large and the velocity 


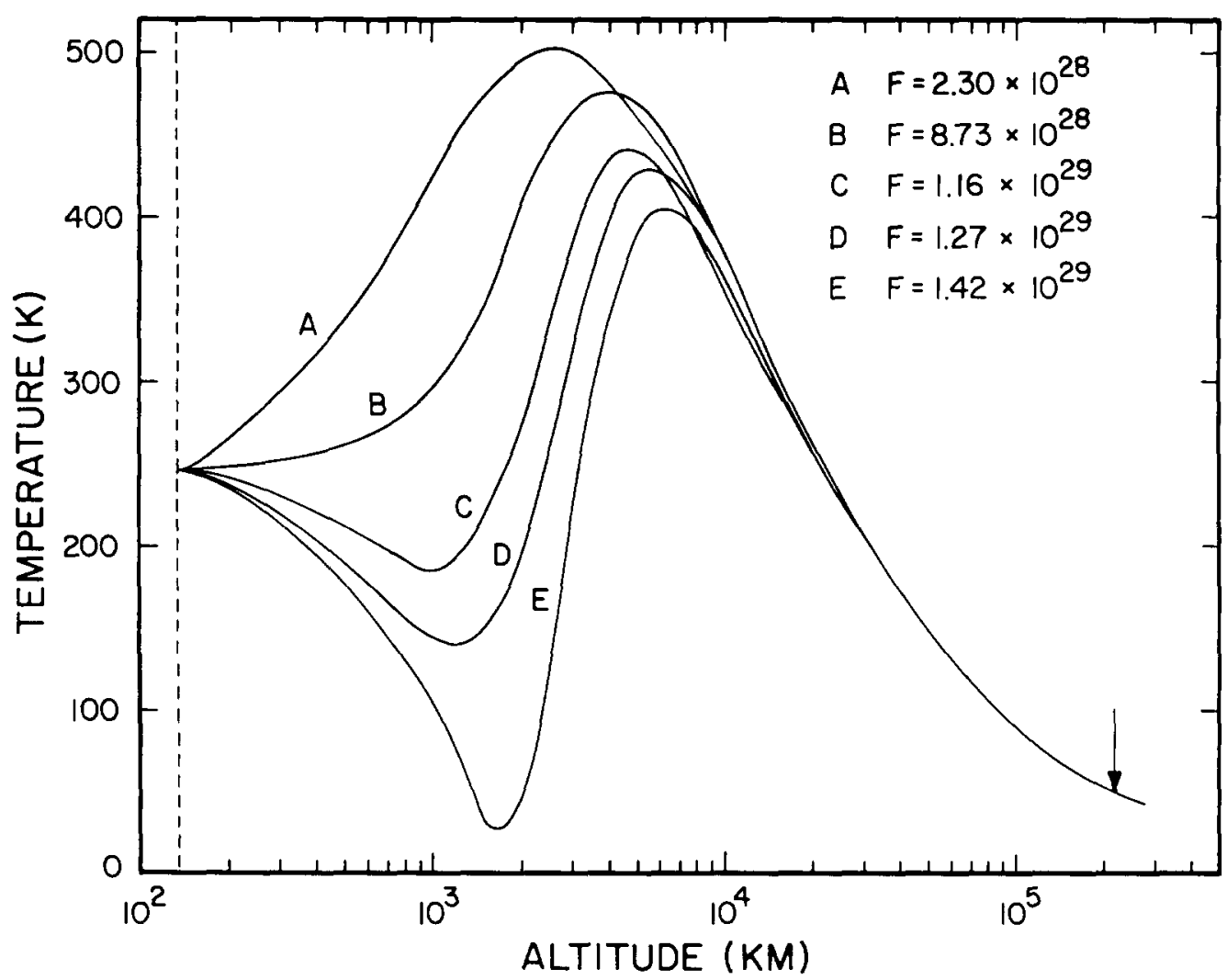

Fig. 4. Numerical solutions to the hydrodynamic equations. Temperature profiles for the escape of a thermosphere of $\mathrm{H}$ atoms from the Earth. Fluxes of particles (per steradian per second) are indicated against each curve. Boundary conditions are zero temperature and pressure as $\lambda \rightarrow 0$ and $T=250^{\circ} \mathrm{K}$ at the lower boundary. The arrow marks the position of the critical point for higher flux curves.

correspondingly small. All the profiles converge above the altitude of the temperature maximum, the density, temperature, and velocity in the outer regions asymptotically approaching a limiting solution as the flux is increased. For that solution, the sonic level is reached at a distance of about $2 \times 10^{5} \mathrm{~km}$ or some 30 planetary radii.

In Fig. 7, curve E from Fig. 4 is plotted together with a comparable temperature profile obtained with the solar euv increased by an order of magnitude. At higher energies the region of maximum absorption moves to lower altitudes and the inner part of the solution contracts somewhat, as might be expected from our previous discussion. The values for the flux parameter $\zeta$ obtained from these two solu- tions are plotted on Fig. 2, where they may be compared with the curve for $\lambda_{0}=30$ obtained from the treatment of the previous section. Considering the approximations involved in that analysis, the agreement seems reasonable.

Recently, Sekiya et al. (1980a) have published some numerical solutions appropriate to a massive, very strongly heated primordial atmosphere. Although their calculations take account of the presence of dust and are not therefore strictly comparable to ours, euv is the most important energy source. We find that solution of Eqs. (16) and (17) gives good agreement with the fluxes obtained by these authors, generally overestimating the escape by a factor between 1 and 3. 


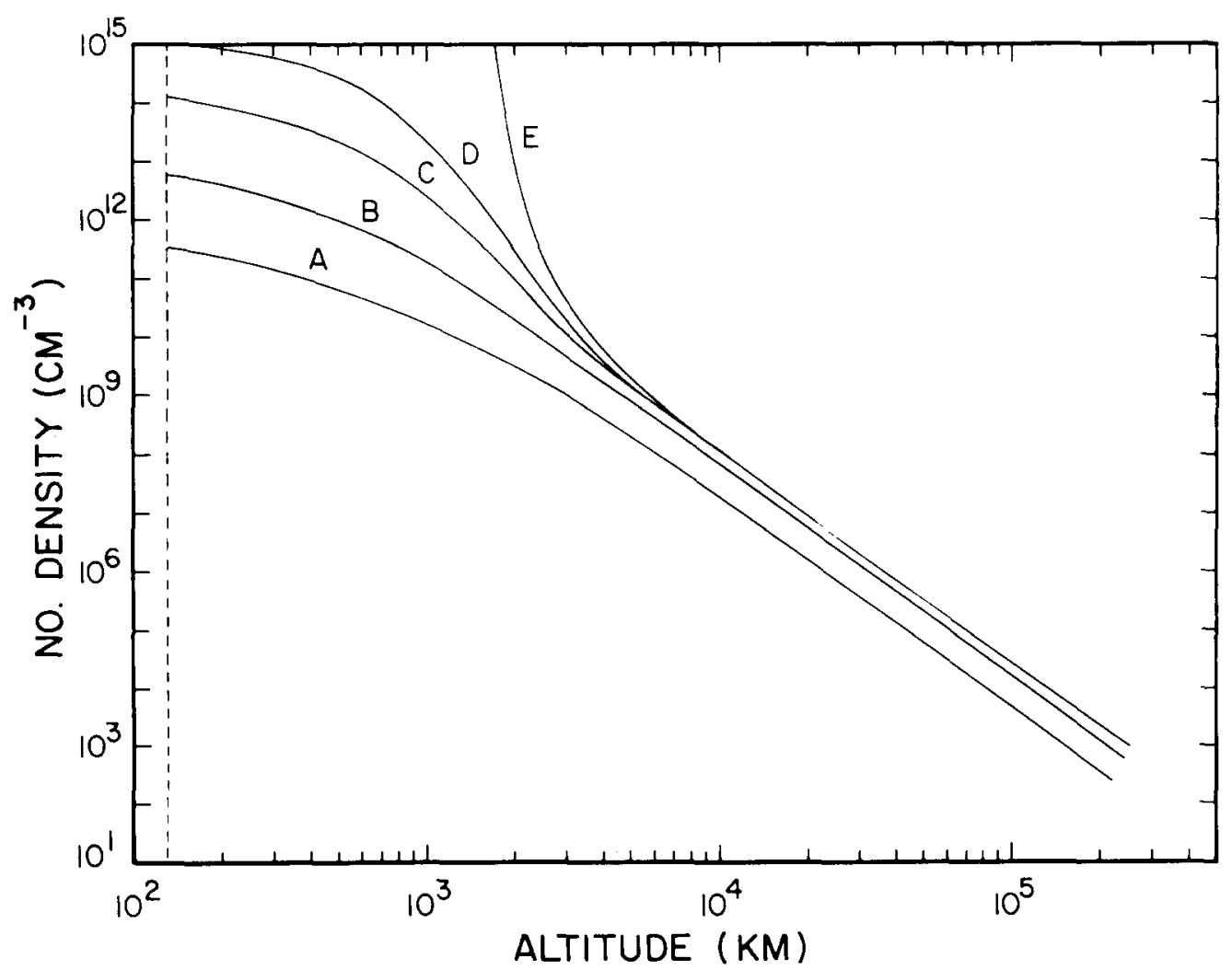

FIG. 5. Number densities corresponding to the temperature profiles of Fig. 4.

\section{PLANETARY APPLICATIONS}

\section{(a) The Early Earth}

As a starting point for our speculations, we assume that the energy in the Sun's euv spectrum was the same during the Hadean (the first billion years of Earth history) as it is today. At altitudes greater than a few hundred kilometers the Earth's thermosphere consisted almost entirely of molecular hydrogen. The temperature near the base of the thermosphere was $250^{\circ} \mathrm{K}$ and the efficiency of euv heating was $30 \%$. The parameters $\lambda_{0}$ and $\beta$ are approximately 60 and $1.1 \times 10^{3}$ respectively for these conditions. Referring to Fig. 2 we find the energy-limited flux parameter $\zeta_{m}$ to be about 0.054 . This corresponds to an escape rate of $2.4 \times 10^{2 y}$ particles $\mathrm{sr}^{-1} \mathrm{sec}^{-1}$ or about $6 \times$ $10^{11} \mathrm{~cm}^{-2} \mathrm{sec}^{-1}$ at the Earth's surface. If this flux had been maintained throughout the Hadean, some $3.3 \times 10^{23} \mathrm{~g}$ of hydrogen would have been lost-about twice the total amount of hydrogen in the crust and ocean of the present-day Earth. We conclude that the potential exists for a considerable oxidation of the planet's crust and atmosphere during this early period.

The above estimate of the energy-limited flux is quite insensitive to a number of our assumptions. For example, it increases by less than a factor of 2 if the temperature at the lower boundary is raised from $250^{\circ} \mathrm{K}$ to $1500^{\circ} \mathrm{K}$, the highest value which is consistent with the assumption of "tight binding." Neither can the flux be greatly increased if we assume that escape occurred simultaneously with accretion; Fig. 8 shows that the escape flux per steradian actually decreases slightly with decreasing mass of the 


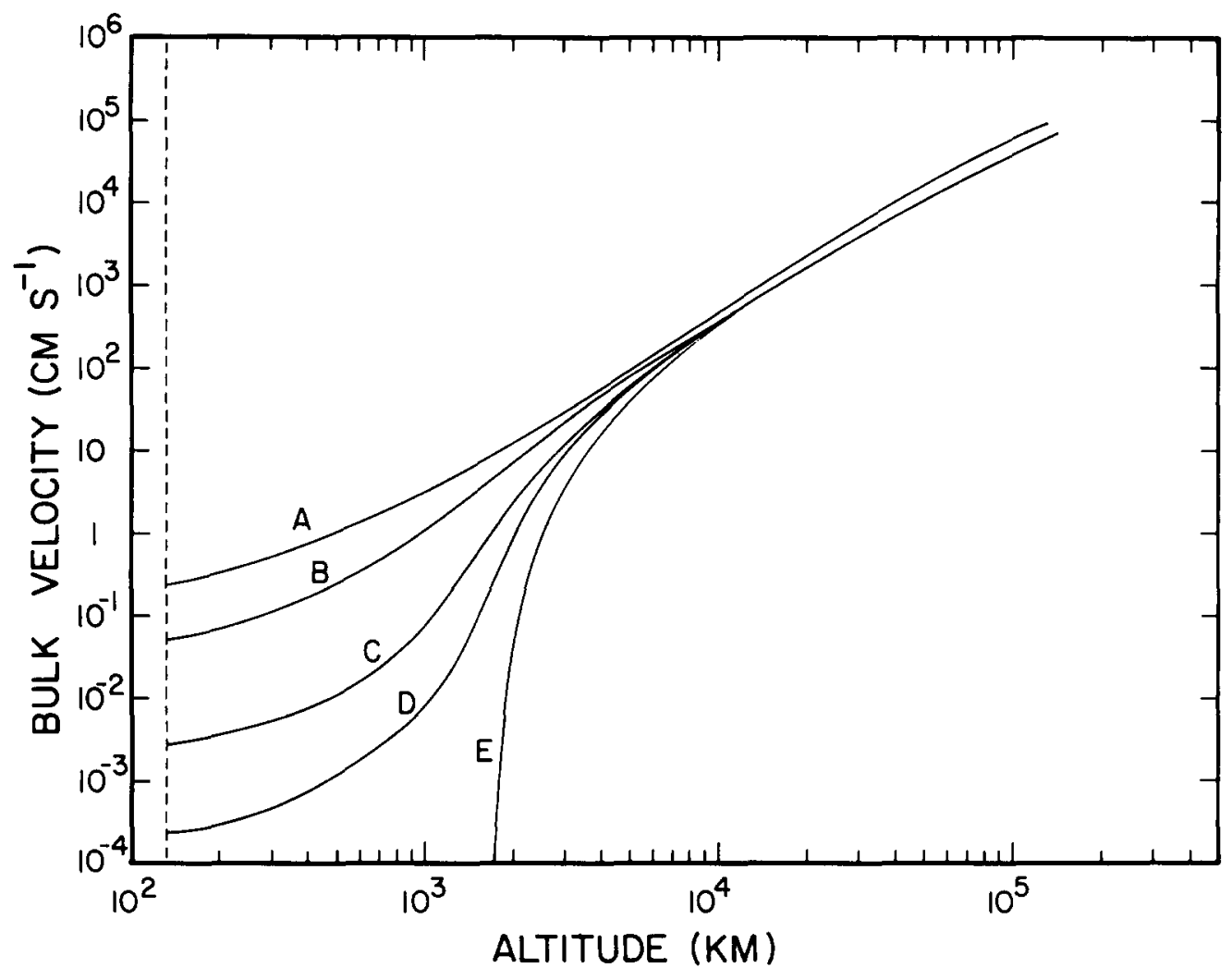

FIG. 6. Bulk velocities for the numerical solutions.

planet, if the density of the accreting body is assumed to be constant. The dependence of the flux on planetary mass is shown for several values of the euv-supplied energy.

If the mixing ratio of total hydrogen in the lower atmosphere is quite small, the escape flux may be limited by the rate at which the hydrogen is able to diffuse through the background, static component of the atmosphere. Hunten $(1973 a, b)$ has derived a simple and quite general expression for this diffusion-limited flux. For a minor constituent,

$$
\begin{aligned}
& F_{1}=n_{i} u_{1} r^{2} \\
& \quad=b(G M / k T)\left(m_{j}-m_{i}\right)\left(n_{i} / n_{j}\right),
\end{aligned}
$$

where $F_{1}$ is the maximum diffusive flux per steradian per second and $b$ is a binary collision parameter of order $10^{19} \mathrm{~cm}^{-1} \mathrm{sec}^{-1}$. Subscripts $i$ and $j$ refer respectively to hydro- gen and the background gas, so that $n_{i} / n_{j}$ is just the $\mathrm{H}_{2}$ mixing ratio. If the background gas is $\mathrm{N}_{2}$, the diffusion-limited flux equals our value of the energy-limited flux when this mixing ratio is about $2.5 \%$. For larger amounts of hydrogen, escape would be energy-limited, while at lower mixing ratios diffusion would be the rate-controlling process.

Equation (18) may also be used to investigate the conditions under which heavier gases are "washed out" of the atmosphere by the escaping hydrogen. We apply the equation to the lower thermosphere, where hydrogen is the major component and the heavier gas is a minor constituent. Subscript $j$ now refers to $\mathrm{H}_{2}$, while $i$ denotes the heavy gas. Dividing (18) by the energy-limited flux, $F=n_{j} u_{j} r^{2}=2.4 \times 10^{29} \mathrm{sr}^{-1} \mathrm{sec}^{-1}$, we obtain the ratio of the diffusion-limited and escape velocities: 


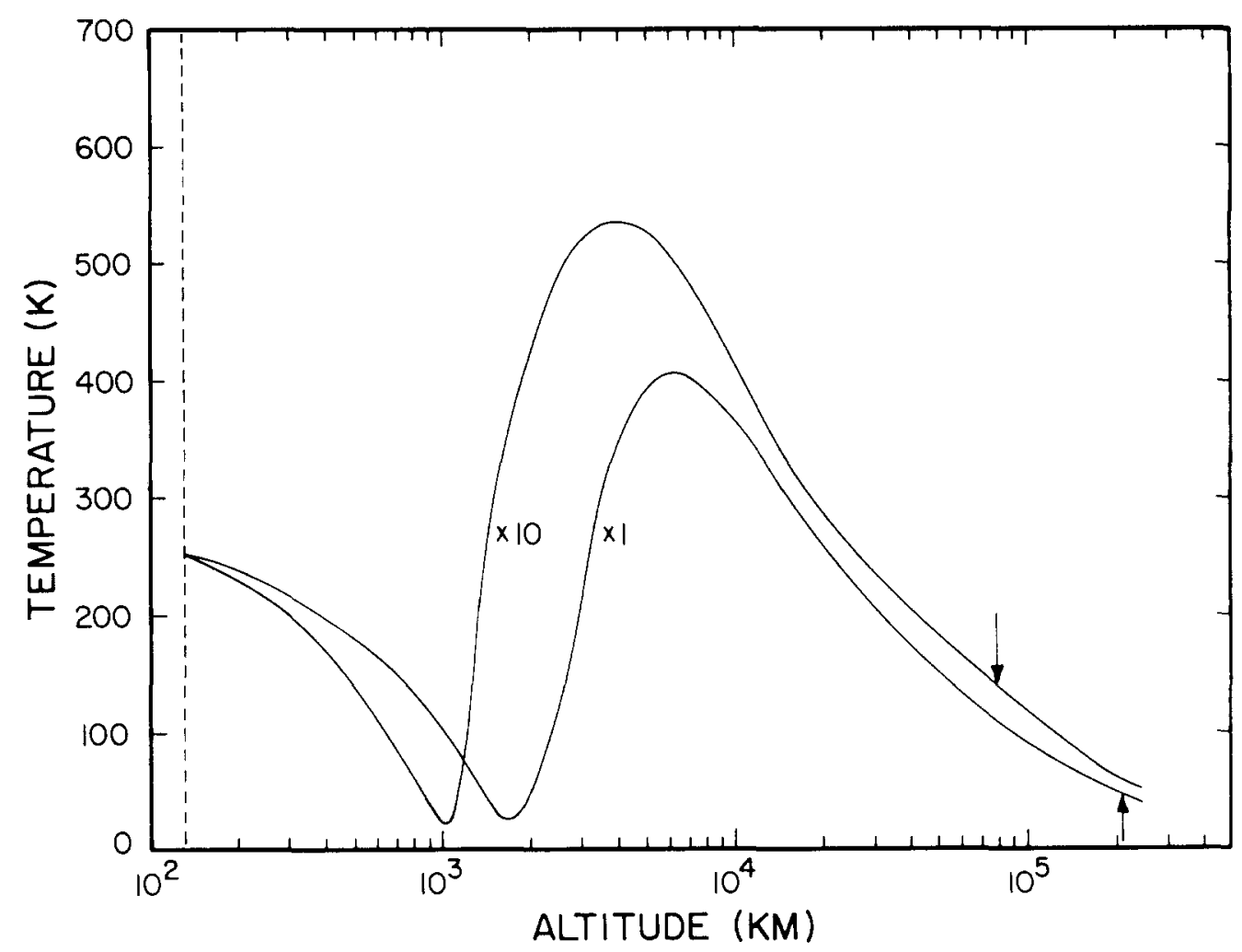

FIG. 7. Numerically obtained temperature profiles close to maximum flux solutions. The $\times 1$ curve was obtained using present-day solar euv flux with a heating efficiency of $15 \%$. For the $\times 10$ curve the energy input was increased by an order of magnitude. Arrows mark the critical levels.

$$
\begin{aligned}
u_{1} / u_{j} & =\left(b G M / F k T_{0}\right)\left(m_{j}-m_{i}\right) \\
& \approx-1.6\left(m_{i} / m_{j}-1\right) .
\end{aligned}
$$

(The ratio is negative because the heavier gas tends to diffuse downwards relative to the escaping hydrogen.) If the absolute value of $u_{1} / u_{j}$ is much greater than one, the heavier gas diffuses downward fast enough to remain in the atmosphere. Conversely, if the absolute value is much less than one, the minor constituent is entrained with the flow of escaping gas. It is apparent that gases as heavy or heavier than atomic nitrogen, for which $m_{i} / m_{j} \geq 7$, are not lost from the planet under our assumptions. However, HD molecules, for which $m_{i} / m_{j}=$ 1.5 , are sufficiently light that they might be washed out of the atmosphere. Escape of copious quantities of hydrogen need not therefore result in a large enrichment of deuterium on the planet.

The question of the "washout" of heavy volatiles has previously been investigated by Hunten (1979) and Sekiya et al. (1980b). These authors considered cases in which the energy available to power escape is many orders of magnitude greater than can be supplied by modern-day solar euv radiation. Hydrogen may then escape rapidly enough to carry with it all the minor constituents regardless of their molecular weights.

The above discussion is relevant to theories for the origin of the Earth which call for the loss of huge amounts of volatiles at an earlier period. For example, the "modified homogeneous accretion theory" (Ringwood, 1975, 1979) requires that the material which condensed to form the Earth con- 


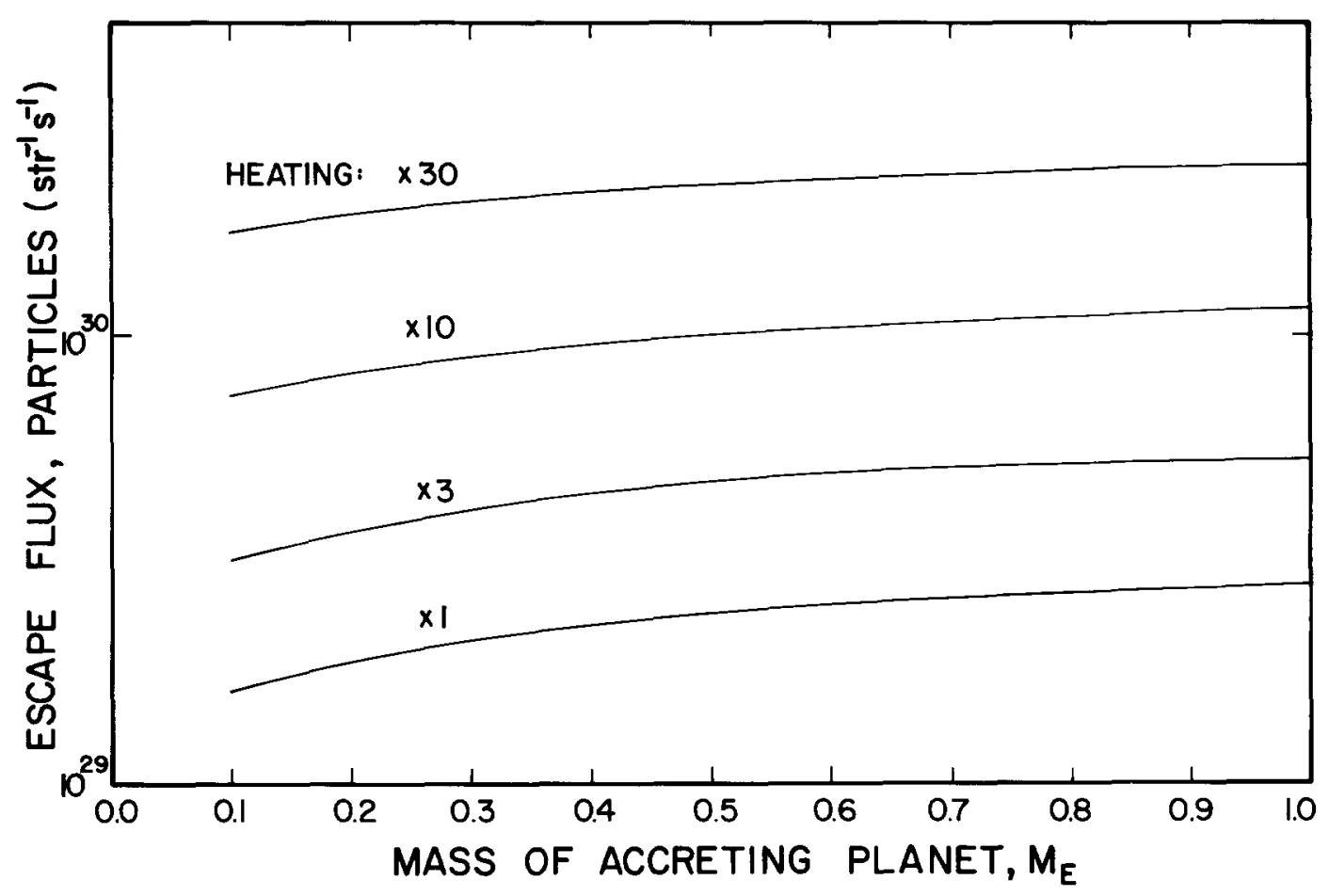

FIG. 8. Energy-limited escape flux of $\mathrm{H}_{2}$ per steradian per second from an accreting Earth, for several values of the euv parameter. $\times 1$ curve refers to present-day solar euv flux, $30 \%$ heating efficiency. The escape rate remains nearly constant regardless of the mass of the accreting planet.

tained roughly $5 \times 10^{24} \mathrm{~g}$ hydrogen, $1.5 \times$ $10^{24} \mathrm{~g}$ nitrogen and $2 \times 10^{25} \mathrm{~g}$ carbon. By contrast the present complements of these elements in the crust, ocean and atmosphere are about $1.9 \times 10^{23} \mathrm{~g}$ of $\mathrm{H}, 5.0 \times$ $10^{21} \mathrm{~g}$ of $\mathrm{N}$ and $1.4 \times 10^{23} \mathrm{~g}$ of $\mathrm{C}$ (Anders and Owen, 1977). Under our assumptions, solar-powered escape accounts for only $10 \%$ of the implied hydrogen loss, while $\mathrm{C}$ and $\mathrm{N}$ do not escape at all.

If such copious escape did indeed occur, it seems unlikely that the energy source was solar euv; that would require the sun to have sustained a level of euv emission several orders of magnitude greater than at present for periods of $10^{8}-10^{9}$ years. A more promising mechanism is strong heating at the base of the atmosphere, due to the release of gravitational energy during the accretion of the planet, as discussed by Ringwood (1975). The very rapid escape requires that the internal energy of the gas be of the same order as its gravitational energy even near the surface; i.e., $\lambda_{0} \sim 2.5$. The implied surface temperature is of order $6000^{\circ} \mathrm{K}$. The temperature may be reduced to $2000^{\circ} \mathrm{K}$ if we are prepared to accept Ringwood's hypotheses that the Earth was rotating rapidly at that time and the atmosphere corotated like a rigid body out to several planetary radii. The high surface temperatures would be maintained only if the planet accreted very rapidly; an accretion time of about $10^{3}$ years is needed if $6000^{\circ} \mathrm{K}$ is the relevant temperature, or $10^{5}$ years if it is $2000^{\circ} \mathrm{K}$ (Jakosky and Ahrens, 1979; Ringwood, 1970). These periods are considerably shorter than the commonly quoted estimate of $10^{8}$ years (Safronov, 1972), but at present the accretion time is poorly known, and a period as short as $10^{5}$ years is not out of the question. The important conclusion is that theories requiring the loss of an early massive atmosphere are 
rather firmly committed to such rapid accretion. They are not consistent with longer time scales, as has been claimed (Ringwood, 1979).

We may also note that Ringwood's theory requires that the Earth ultimately retained $1 \%$ or less of its original nitrogen and carbon complement. Since these elements cannot have escaped once the temperature dropped below $\sim 2000^{\circ} \mathrm{K}$, it follows that less than $1 \%$ of the volatiles were brought in during the final stages, as the accretion rate declined towards zero. A very abrupt cessation of accretion is implied, in contrast to the pronounced tail-off which might be expected as the last of the planetesimals were swept up by the newly formed planet. We conclude that the loss of large quantities of heavy volatiles requires a highly specific accretion history for the planets.

\section{(b) Venus}

Most of the above discussion for the Earth applies equally to Venus. For the same conditions as before, we calculate an energy-limited $\mathrm{H}_{2}$ flux of $1.1 \times 10^{12} \mathrm{~cm}^{-2}$ $\sec ^{-1}$ at the planet's surface. Loss of the equivalent of an ocean of hydrogen would occur in a mere $280 \mathrm{my}$ at this rate, so that the water-rich atmosphere which would have resulted from a Cytherean "runaway greenhouse" may have lasted for only a small fraction of the planet's history. As on Earth, the hydrogen would not escape so rapidly that significant quantities of heavy volatiles would be lost from the planet, so the oxygen released by dissociation of the water must still be on Venus; it may have reacted with the surface (Walker, 1975) or with reduced gases in the atmosphere (Dayhoff et al., 1967).

Just as on Earth, on Venus we need not expect deuterium to have been left behind by the rapid escape of hydrogen. The escape of oceans of water does not, therefore, imply a huge residue of deuterium on the planet. Such a residue was at one time proposed to explain the two-component Ly- $\alpha$ emission observed by Mariner 5 (McElroy and Hunten, 1969), but subsequent observations disproved this hypothesis (Wallace et al., 1971).

However, if the low abundance of hydrogen now observed is attributed exclusively to escape of the gas, some enrichment of deuterium is bound to have occurred. From Eq. (19), it can be shown that the escape velocity of hydrogen is insufficient to remove deuterium from the atmosphere once the flux (referenced to the planet's surface) drops below about $4 \times 10^{11} \mathrm{~cm}^{-2} \mathrm{sec}^{-1}$. Using Eq. (18), we find that diffusion through the dense $\mathrm{CO}_{2}$ component of the atmosphere limits the flux to this value when the mixing ratio of hydrogen declines to about $2 \%$. But the present-day content of total hydrogen in the Venusian atmosphere is, at most, about 0.1\% (Oyama et al., 1979). Therefore, the escape of the last remnant of hydrogen would have enriched by at least a factor of 20 the present-day ratio of deuterium to hydrogen. Measurements of the Pioneer Venus neutral mass spectrometer, while not conclusive on this issue, indicate that no very large difference exists between the $\mathrm{D} / \mathrm{H}$ ratios of the Earth and Venus (Hoffman et al., 1980). It seems most likely, therefore, that the last few percent of the "lost" hydrogen on Venus did not escape the planet, though the mechanism by which it was removed from the atmosphere remains an unsolved problem. Of course it is possible that, as Lewis $(1969,1980)$ has maintained, Venus was all but devoid of water from the time of its formation and no runaway greenhouse event ever occurred.

In conclusion, this work illustrates that it is important to consider the energy budget when dealing with the escape of large quantities of gases. After the accretion of the planets, the energy available to power escape processes is rather limited, probably being confined to solar euv heating. The dynamics of the escape process allow only a limited amount of this energy to be intercepted and converted into escape energy. During accretion, some gravitational energy may be available to power escape, but 
only if the time scale for formation of the planet is very short. Energy considerations do not rule out the loss of considerable quantities of hydrogen and deuterium from the primordial Earth and Venus, but removal of heavier elements is much less likely to have occurred.

APPENDIX: DERIVATION OF Eqs. (16) AND (17), AND JUSTIFICATION OF ASSUMPTIONS

We first show that the assumption that the level of unity optical depth is below the sonic level implies relation (11):

$$
\lambda_{1} / \tau_{1}>2 .
$$

We then derive Eqs. (16) and (17). Finally we examine the validity of our assumptions.

Above $r_{1}$, the energy flow term, $\epsilon$ in (9), is positive and equal to $\epsilon_{\infty}$. In this region, Eqs. (8) and (9) are identical in form to those used in the 'study of the solar wind. Since the gas is continuing to expand against gravity and must in addition carry some energy from $r_{1}$ to infinity by conduction, the temperature is declining, meaning $d \tau / d \lambda$ is positive. At the sonic level where $\tau=\Psi$, the right side of the momentum Eq. (8) must reduce to zero;

$$
1-2 \tau_{\mathrm{s}} / \lambda_{\mathrm{s}}-(d \tau / d \lambda)_{\mathrm{s}}=0 .
$$

(This is a necessary condition if $d \Psi / d \lambda$ is to remain finite). Since $d \tau / d \lambda$ is positive,

$$
\lambda_{\mathrm{s}} / \tau_{\mathrm{s}}>2 \text {. }
$$

Furthermore, it is characteristic of this type of solution that the ratio $\lambda / \tau$ declines monotonically with increasing distance (see, e.g., Parker, 1964b; Noble and Scarf, 1963). Since $r_{1}$ lies inside the sonic level, we may infer (A1) from (A3).

We now derive Eqs. (16) and (17) from (15). Direct integration of (15) subject to the lower boundary condition $\tau\left(\lambda_{0}\right)=1$ gives:

$$
\begin{aligned}
{[2 /(1+s) \zeta]\left(1-\tau^{s+1}\right) } & \\
& =\lambda_{0}{ }^{2}-\lambda^{2}-2\left\{\beta / \zeta \lambda_{1}{ }^{2}-\epsilon_{\infty}\right\}\left(\lambda_{0}-\lambda\right) .
\end{aligned}
$$

When $\zeta=\zeta_{\mathrm{m}}$, (the maximum possible flux), $\tau=0$ at $\lambda=\beta /\left(\zeta_{\mathrm{m}} \lambda_{1}{ }^{2}\right)-\epsilon_{\infty}$, the position of the temperature minimum. Applying this condition we obtain a relation between the unknowns $\zeta_{m}$ and $\lambda_{1}$;

$$
\begin{aligned}
{\left[2 /(1+s) \zeta_{m}\right]^{1 / 2} } & =\lambda_{0}-\beta /\left(\zeta_{m} \lambda_{1}{ }^{2}\right)+\epsilon_{\infty} .
\end{aligned}
$$

A second relation may be derived as follows. We obtain an estimate of the temperature near the level of unity optical depth by integrating Eq. (15) from the temperature minimum to $\lambda_{1}$. Close to $\lambda_{1}$, the temperature may be high enough that it is no longer accurate to neglect the term $5 \tau / 2$ in the full energy Eq. (14). The approximate solution therefore gives only a lower limit for $\tau_{1}$. Performing this integration yields

$\left[2 /(1+s) \zeta_{m}\right]^{1 / 2} \tau_{1}^{(1+s) / 2}$

$$
>\beta /\left(\zeta_{\mathrm{m}} \lambda_{1}{ }^{2}\right)-\epsilon_{\infty}-\lambda_{1} .
$$

$\tau_{1}$ can be eliminated from this expression by using relation (A1), and the quantity $\beta /\left(\zeta_{m} \lambda_{1}{ }^{2}\right)-\epsilon_{\infty}$ can be replaced by substituting from (A4):

$$
\begin{aligned}
& {\left[2 /(1+s) \zeta_{m}\right]^{1 / 2} \cdot\left(\lambda_{1} / 2\right)^{(1+s) / 2}} \\
& \quad=\lambda_{0}-\left[2 /(1+s) \zeta_{m}\right]^{1 / 2}-\lambda_{1}+\epsilon^{\prime}
\end{aligned}
$$

where we have added the arbitrary positive quantity $\epsilon^{\prime}$ to the right side in order to make the relation an equality.

Finally, some rearrangement of (A4) and (A5) yields the explicit equations for $\lambda_{1}$ and $\zeta_{\mathrm{m}}$ used in the text:

$$
\begin{aligned}
\lambda_{1}=\left\{\frac{\beta}{\zeta_{m}}\right. & {\left[\lambda_{0}\right.} \\
& \left.\left.-\left[\frac{2}{(1+s) \zeta_{m}}\right]^{1 / 2}+\epsilon_{\infty}\right]^{-1}\right\}^{1 / 2}, \\
\zeta_{m} & =\frac{2}{1+s}\left\{\frac{\left(\lambda_{1} / 2\right)^{(1+s) / 2}+1}{\lambda_{0}-\lambda_{1}+\epsilon^{\prime}}\right\}^{2} .
\end{aligned}
$$

One may show that neglecting $\epsilon_{\infty}$ and $\epsilon^{\prime}$ leads to an overestimate of $\zeta_{m}$ as follows. Over the physical range of variables, $\partial \lambda_{1} / \partial \zeta_{m}$ and $\partial \lambda_{1} / \partial \epsilon_{\infty}$ obtained from Eq (A6) are both negative. Similarly, $\partial \zeta_{\mathrm{m}} / \partial \epsilon^{\prime}$ found from (A7) is negative but $\partial \zeta_{m} / \partial \lambda_{1}$ is positive. For either $\epsilon_{\infty}$ or $\epsilon^{\prime}$, the total derivative $d \zeta_{\mathrm{m}} / d \epsilon$, given by 


$$
\frac{d \zeta_{\mathrm{m}}}{d \epsilon}=\frac{\partial \zeta_{\mathrm{m}} / \partial \epsilon+\left(\partial \zeta_{\mathrm{m}} / \partial \lambda_{1}\right)\left(\partial \lambda_{1} / \partial \epsilon\right)}{1-\left(\partial \zeta_{\mathrm{m}} / \partial \lambda_{1}\right)\left(\partial \lambda_{1} / \partial \zeta_{m}\right)}
$$

is then negative. Since $\epsilon^{\prime}$ and $\epsilon_{\infty}$ are positive definite, fluxes obtained with $\epsilon^{\prime}=\epsilon_{\infty}=0$ must be greater than those obtained with any other values.

\section{EXAMINATION OF ASSUMPTIONS}

\section{(a) $r_{1}$ Lies below the Sonic Level}

The conditions under which this assumption is valid may be investigated by obtaining an upper limit on $\Psi_{1}$, the velocity variable at unity optical depth. If the atmosphere were isothermal and static, the condition for unity optical depth would be

$$
n_{1} H_{1} \sigma=1 \text {, }
$$

where $\sigma$ is an appropriately averaged cross section to euv and

$$
H_{1}=k \tau_{1} T_{0} r_{1}^{2} / G M m
$$

is the equilibrium scale height at $r_{1}$. Because the gas is accelerating and the temperature is declining beyond $r_{1}$, the actual scale height is less than the equilibrium value, thus

$$
n_{1} H_{1} \sigma>1 \text {. }
$$

Using the definition of $\zeta$ [Eq. (12) in the text] with $F=n_{1} u_{1} r_{1}{ }^{2}$, we may write $n_{1}$ as

$$
n_{1}=\kappa_{0} G M m \zeta / k^{2} T_{0} r_{1}{ }^{2} u_{1}
$$

Substituting (A 10) and (A8) in (A9) there results

$$
\kappa_{0} \tau_{1} \zeta \sigma / k u_{1}>1
$$

from which, since $\Psi_{1}=m u_{1}^{2} / k T_{0}$, we obtain

$$
\Psi_{1}<\left(m / k^{3} T_{0}\right)\left(\kappa_{0} \tau_{1} \zeta \sigma\right)^{2} .
$$

Now the cross section of hydrogen molecules to euv is, at its maximum, $10^{-17} \mathrm{~cm}^{2}$. We restrict our attention to cases where $\zeta$ is 10 or less, and take $T_{0}=1000^{\circ} \mathrm{K}$, in which case $\kappa_{0}=4.45 \times 10^{4}$ ergs $\mathrm{cm}^{-1} \mathrm{sec}^{-1}{ }^{\circ} \mathrm{K}^{-1}$ (Hanley et al., 1970). This choice, which is made deliberately to yield a generous upper limit on $\Psi_{1}$, gives

$$
\Psi_{1}<0.025 \tau_{1}{ }^{2}
$$

For all values of $\tau_{1}$ less than $40, \Psi_{1}$ is therefore less than $\tau_{1}$. We know that $\Psi<\tau$ everywhere below $r_{\mathrm{s}}$ and $\Psi>\tau$ everywhere above $r_{\mathrm{s}}$, so it follows that $r_{1}<r_{\mathrm{s}}$ for these conditions. We conclude that the assumption is justified over the range of parameters utilized in the text, but that it may break down if the atmosphere is so strongly heated as to allow very high escape rates simultaneously with high temperatures in the thermosphere.

\section{(b) Boundary Conditions at Infinity}

In the text it is assumed that the pressure at infinity is zero and the gas accordingly expands supersonically. In reality the pressure at large distances is set by conditions in the interplanetary medium. However, the effect of nonzero pressures at infinity can only be to slow the escape of the gas. Since our purpose is to obtain upper limits for the escape, the assumption does not affect the validity of the results.

The analysis does not assume any specific value for the temperature at infinity. It does, however, assume that energy deposited above $r_{1}$ cannot be conducted downward into the inner thermosphere. At low escape rates this requires that a temperature maximum exists near the position of unity optical depth, so that temperatures decline above that region. At high loss rates conduction becomes progressively less able to redistribute the absorbed energy; under such conditions the temperature profile above the minimum is largely irrelevant to the problem. The positions of unity optical depth and the temperature minimum must then be essentially coincident.

\section{(c) Convective Instability}

An assumption which is implicit in the analysis is that temperatures in the innermost region never decline so rapidly as to be superadiabatic. At high escape rates this is not necessarily true. A portion of the 
temperature profile should then be replaced by an adiabat to take account of convective motions. A temperature minimum still results and the situation may be analyzed by a technique very similar to the convectively stable case. One obtains upper limits on the escape flux which never exceed by more than a factor of 2.5 the values given in Fig. 2. For the sake of brevity a detailed description of the convective case is not given here.

\section{ACKNOWLEDGMENTS}

This research was supported in part by NASA Grants NAS2-9125, NAS2-9126, and NAGW-176. The authors thank J. F. Kasting for helpful discussion on this subject.

\section{REFERENCES}

ANDERS, E., AND OWEN, T. (1977). Mars and Earth: Origin and abundance of volatiles. Science 198, 453465.

Banks, P. M., and Kockarts, G. (1973). Aeronomy. Academic Press, New York.

Chamberlain, J. W. (1961). Interplanetary gas. III. A hydrodynamic model of the corona, Astrophys. $J$. 133, 675-687.

Chamberlain, J. W. (1962). Upper atmospheres of the planets. Astrophys. $J$. 136, 582-593.

Chamberlain, J. W. (1963). Planetary coronae and atmospheric evaporation. Planet. Space Sci. 11, 901-960.

DAYHOFF, M. O., Eck, R., LIPPINCOTT, E. R., AND SaGan, C. (1967). Venus: Atmospheric evolution. Science 155, 556-558.

Gross, S. H. (1972). On the exospheric temperature of hydrogen-dominated planetary atmospheres. $J$. Atmos. Sci. 29, 214-218.

Gross, S. H. (1974). The atmospheres of Titan and the Galilean satellites. J. Atmos. Sci. 31, 1413-1430.

HANLEY, H. J. M., MCCARTY, R. D., AND INTERMAN, H. (1970). The viscosity and thermal conductivity of dilute gaseous hydrogen from 15 to $500 \mathrm{~K}$. J. Res. Nat. Bur. Stand. Sect. A 74, 331-350.

Hoffman, J. H., Hodges, R. R., Donahue, T. M. AND MCELROY, M. B. (1980). Composition of the lower atmosphere of Venus from the Pioneer Venus mass spectrometer. J. Geophys. Res. 85, 78827890.

Holland, H. D. (1962). A model for the evolution of the Earth's atmosphere. In Petrologic Studies: $A$ Volume in Honor of A. F. Buddington, (A. E. J. Engel, H. L. James, and B. F. Leonard, Eds.). Geol. Soc. Amer., Boulder, Colo.
Hunten, D. M. (1973a). The escape of $\mathrm{H}_{2}$ from Titan. J. Atmos. Sci. 30, 726-732.

Hunten, D. M. (1973b). The escape of light gases from planetary atmospheres. J. Atmos. Sci. 30, $1481-1494$.

Hunten, D. M. (1979). Capture of Phobos and Deimos by protoatmospheric drag. Icarus 37, 113-123.

Hunten, D. M., And Donahue, T. M. (1976). Hydrogen loss from the terrestrial planets. Annu. Rev. Earth Planet. Sci. 4, 265-292.

INGERSOLL, A. P. (1969). The runaway greenhouse: A history of water on Venus. J. Atmos. Sci. 26, 11911197.

Jakosky, B. M., and Ahrens, T. S. (1979). The history of an atmosphere of impact origin. Proc. Lunar Planet. Sci. Conf. 10th, 2727-2739.

Jeans, J. H. (1925). The Dynamical Theory of Gases. 4th ed. Cambridge Univ. Press. London/New York.

LEwIS, J. S. (1969). Venus: Atmospheric and lithospheric composition. lcarus 11, 367-385.

Lewis, J. S. ANd Kreimendahl, F. A. (1980). Oxidation state of the atmosphere and crust of Venus from Pioneer Venus results. Icarus 42, 330-337.

MCElroy, M. B. (1968). The upper atmosphere of Venus. J. Geophys. Res. 74, 29-41.

McElroy, M. B. (1974). Comment. In The Atmosphere of Venus (J. E. Hansen, Ed.), p. 172. NASA publication SP-382, NASA, Washington, D.C.

MCElroy, M. B., AND Hunten, D. M. (1969). The ratio of deuterium to hydrogen in the Venus atmosphere. J. Atmos. Sci. 28, 879-884.

Noble, L. M., and Scarf, F. L. (1963). Conductive heating of the solar wind. Astrophys. J. 133, 1169 1181 .

ÖPIK, E. J. (1963). Selective escape of gases. Geophys. J. Roy. Astron. Soc. 7, 490-509.

Oyama, V. I., Carle, G. C., Woeller, F., and Pollack, J. B. (1974). Venus lower atmosphere composition: Analysis by gas chromatography. Science 203, 802-804.

PARKer, E. N. (1963). Interplanetary Dynamical Processes. Wiley, New York.

Parker, E. N. (1964a). Dynamical properties of stellar coronas and stellar winds. I. Integration of the momentum equation. Astrophys. J. 139, 72-92.

PARKer, E. N. (1964b). Dynamical properties of stellar coronas and stellar winds. II. Integration of the heat flow equation. Astrophys. J. 134, 93-122.

Pollack, J. B. (1971). A non-grey calculation of the runaway greenhouse: Implications for Venus' past and present. Icarus 14, 295-306.

Pollack, J. B., ANd Black, D. C. (1979). Implications of the gas compositional measurements of Pioneer Venus for the origin of planetary atmospheres. Science 205, 56-59.

Pollack, J. B., AND YUng, Y. L. (1980). Origin and evolution of planetary atmospheres. Annu. Rev'. Earth Planet. Sci. 8, 425-487. 
Ringwood, A. E. (1970). Origin of the Moon: The precipitation hypothesis. Earth Planet. Sci. Lett. 8, $131-140$.

Ringwood, A. E. (1975). Composition and Petrology of the Earth's Mantle. McGraw-Hill, New York.

RiNGwood, A. E. (1979). Origin of the Earth and Moon. Springer-Verlag, Berlin/New York.

Sekiya, M., Nakazawa, K., and Hayashi, C. (1980a). Dissipation of the primoridal terrestrial atmosphere due to irradiation of the solar euv. Prog. Theor. Phys. 64, 1968-1985.

Sekiya, M., Nakazawa, K., and Hayashi, C. $(1980 \mathrm{~b})$. Dissipation of the rare gases in the primoridal Earth's atmosphere. Earth Planet. Sci. Lett. 50, 197-201.
ShKLovskII, L. S. (1951). On the possibility of explaining the difference in chemical composition of the Earth and the Sun by thermal dissipation of light gases. Astron. Zh. 28, 234-243.

WALKER, J. C. G. (1975). Evolution of the atmosphere of Venus. J. Atmos. Sci. 32, 1248-1256.

WALker, J. C. G. (1977). Evolution of the Atmosphere. MacMillan, New York.

Wallace, L., Stuart, F. E., Nagel, R. H., and LARSON, M. D. (1971). A search for deuterium on Venus. Astrophys. J. 168, 189-191.

WANG, Y. C., AND Chang, C. C. (1965). An inviscid model of the solar wind. J. Geophys. Res. 70, 4175 4180 . 